\title{
Evaluating the work of ethical review committees: an observation and a suggestion
}

\author{
Timothy Harding and Marinette Ummel University Institute of Legal Medicine, Geneva
}

\section{Authors' abstract}

Eight research protocols which had previously been approved by Ethical Research Committees (ERCs) were reviewed in simulated review committees set up during a symposium on medical ethics. Only three protocols were considered to provide fully adequate information to allow ethical review and only one protocol was thought to provide sufficient guarantees on the ethical issues raised by the proposed research. For five other protocols additional safeguards were considered necessary, in particular covering the problem of informed consent. Two protocols were considered to raise unresolvable ethical issues.

This artificial exercise does not establish that review by $E R C$ s is ineffective. It does highlight the lack of objective criteria in ethical review. Peer review by exchange of protocols between ERCs could assist in increasing the consistency in the application of ethical standards.

\section{Introduction}

The work of ethical review committees (ERCs) can be seen as meeting three separate needs (1):

1. to maintain recognised ethical standards by submitting all health-related research involving human subjects to an independent review, with the possibility of strengthening safeguards concerning information to subjects, consent, confidentiality and the degree of risk;

2. to allow some degree of flexibility in interpreting ethical codes so as to permit research on unconscious patients, mentally ill people, children and other groups for whom informed consent by the research subject is not always possible,

3. to provide interpretations of existing ethical codes in technologically innovative situations.

In Switzerland, a federal state in which the cantons retain considerable autonomy, the development of ERCs has been stimulated by two distinct processes. The Swiss Academy of Medical Sciences, a non-

\section{Key words}

Ethical review committees; human experimentation; informed consent; confidentiality; Switzerland; ethical committees; institutional review boards (IRBs). governmental consultative body, has issued directives concerning medical research in which it is recommended that ERCs be established in hospitals and universities and that all research projects involving human subjects be submitted for review. The academy has itself created a central committee on medical ethics which is in contact with ERCs at institutional level. The other stimulus has been legal; although no federal law on human research exists, many cantons have enacted legislation. Cantonal laws on research involving human subjects are varied; for example there is divergence over the need for written confirmation of a research subject's consent. The legislation in several cantons makes reference to the academy's directiveso specifying that experiments involving human subjects must conform to these requirements, including review by ERC. This amalgam of legal and independent professional controls raises considerable problems in assigning responsibilities and in interpretation of the regulations. In other cantons, the establishment of ERCs is required without reference to the academy's directives. Thus directly or indirectly ERCs have acquired a legal basis in Switzerland. Over 40 ERCs now function in the country. Their membership, their role and their method of work vary considerably.

But how effective are such committees in ensuring the application of adequate ethical safeguards and standards in medical research? In a study of attitudes to such committees by their members, Allen and Waters (2) showed a high level of agreement on the need for such committees but also a feeling that the system could be improved, especially by training for committee members. Lewis (3) has pointed out several drawbacks of ERCs, particularly the diminution of the individual responsibility assumed by the researcher once a project has been approved by a committee.

In the face of considerable variation in the ways in which ERCs are composed and carry out their work, comparative studies would certainly be useful and one of us (MU) is completing a detailed survey of ERCs in Switzerland. Meanwhile, we believe that the results of a small-scale exercise, in which eight research projects were reviewed by ad hoc groups during a symposium on medical ethics, composed largely of ERC members are of some interest in evaluating the work of ERCs. A comparison can be made between the views expressed 
by the ad hoc groups and the original review committee decisions.

\section{Method}

A symposium on medical ethics organised by the Faculty of Medicine of the University of Geneva provided the setting. Participants were drawn from the medical faculties of the Universities of Geneva, Zürich and Lausanne as well as from the Swiss Academy of Medical Sciences. The majority were medical practitioners with a smaller number of nurses, theologians and other professions. All had served or were still members of ERCs at departmental, institutional or faculty level.

Four groups were formed, each with ten members, two of whom functioned as moderator and rapporteur respectively. Three of the groups were asked to function as ERCs in a simulation exercise. (The fourth group considered questions concerning the composition and function of ethical research committees: their findings are not included in this paper.)

Each group met for one and a half hours. Three research protocols were submitted to each group. The protocols were selected from amongst those which had already been considered by various ERCs functioning within the three medical faculties taking part in the symposium. Selection was made to include projects from different medical disciplines (internal medicine, surgery, oncology, cardiology, toxicology, pharmacology, psychiatry, gynaecology) and involving different categories of research subjects (healthy volunteers, the researchers themselves, children, families of patients, patients in intensive care, outpatients). The groups were informed neither from which university the research protocol had been selected nor of the decision of the ERC.

At the end of their deliberations, the rapporteur of each group gave a summary report of the conclusions to a plenary session of the symposium. Subsequently, the rapporteurs submitted a more detailed written report.

\section{Results}

Groups I and II were each able to consider and reach conclusions about three research protocols. Group III found the time allotted insufficient and reached conclusions on only two protocols. Results are therefore available on eight protocols. The assessments given below were those of the groups in the simulation exercise. In reporting them we do not imply that their judgements were correct and those of the original ERCs incorrect.

All eight research protocols had been found acceptable by institutional ERCs. In some instances modifications had been demanded to the original protocol. In every case, the protocol in its final, accepted form with all explanatory notes and exchange of correspondence was submitted to the groups during the simulation exercise.

Only three protocols were thought to provide fully adequate information: ie on the nature of the proposed research and the ways of dealing with various ethical problems. In four further protocols, information was barely adequate to allow the groups to reach conclusions: further information, especially on ethical issues would have been useful. One protocol was thought to give inadequate information to allow consideration by an ethical committee.

Each protocol was thought to raise at least two ethical issues. On average 4.2 issues were noted per protocol. Less than half of the issues ( 15 out of 34 ) were considered to have been satisfactorily dealt with in the research protocol. Of the 19 remaining issues, 16 could have been resolved by conditions laid down by an ERC, provided that these were accepted and observed by the researchers.

Three ethical issues were thought to be so serious and problematic that they would have led to rejection of the research on ethical grounds. One protocol presented two such problems.

Informed consent was a potential problem in every project, and adequately dealt with in only one protocol. For three projects, the groups would have required precise, written information for research subjects or their relatives. In the case of seriously ill patients, the problem of giving adequate information for informed consent when the prognosis was bad was thought to be specially difficult and insufficiently considered by the researchers in their protocols. In two cases an independent medical opinion on each potential research subject would have been required as a condition of approval. In the case of proposed family interviews in a crisis situation for research purposes only (following a suicidal attempt by an adolescent), the group thought that informed consent could not reasonably be obtained.

The degree of risk for research subjects and their subjective discomfort, were thought to raise problems in six of the protocols, but in all cases the issues had been adequately dealt with or additional safeguards had been suggested.

The usefulness of the research was doubted in four out of eight projects. In one case this issue led to outright rejection: the treatment under study was thought to have no scientific basis and the research design was such that no conclusions on efficacy could have been reached. The results, it was thought, would have served only for publicity for the drug manufacturer and not for further scientific investigation. One other problem concerning 'usefulness' for which further assurances would have been required, was the possible 'hidden' use of a research procedure in the case of a treatment (plasmapharesis) which could provide material (immune complexes) useful in parallel non-therapeutic research. The group would have required that the research team undertaking the therapeutic research was not directly involved in any research on the material obtained. The same research project was thought to raise unresolved issues of follow-up 


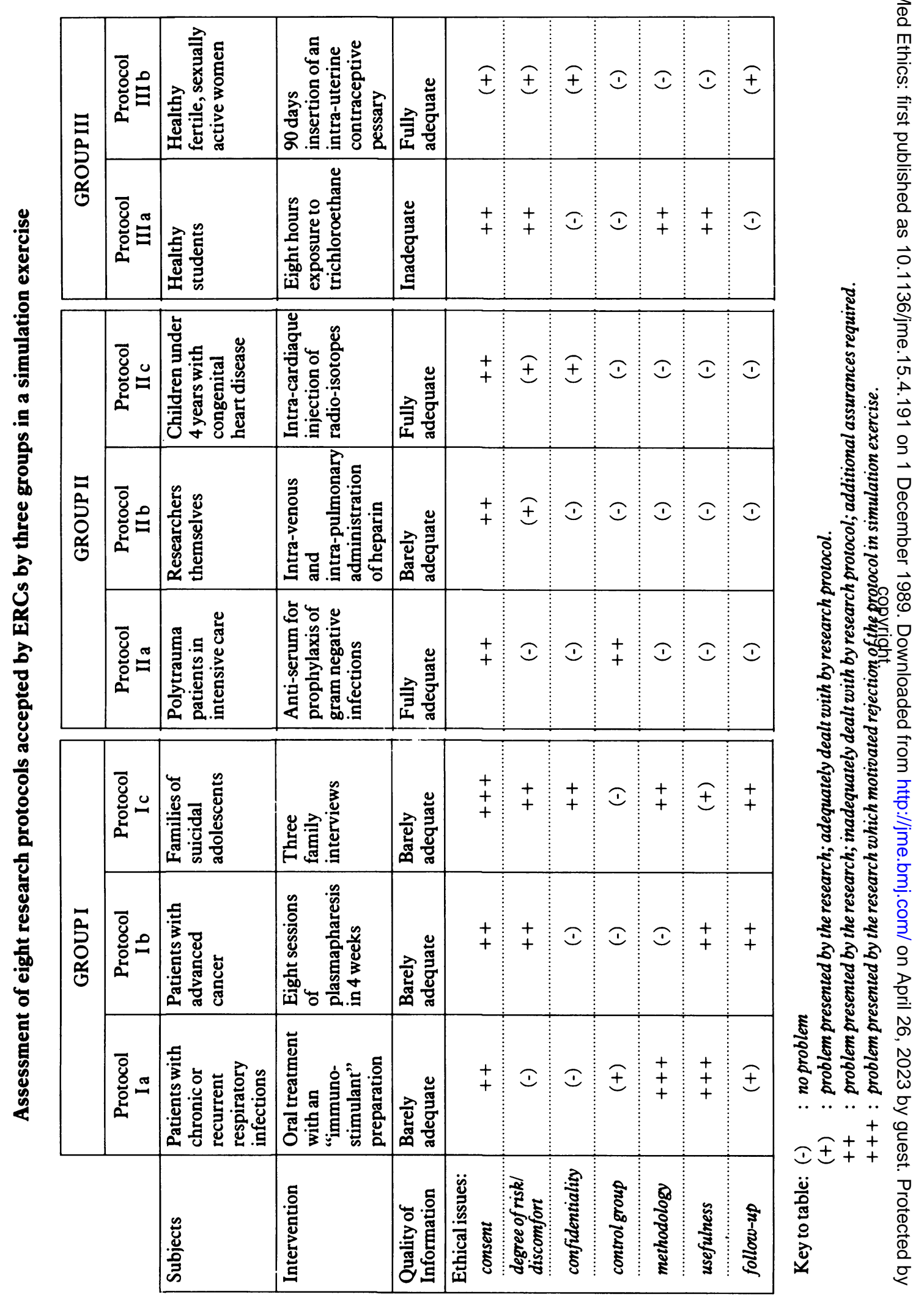


treatment. This was also the case in the research on the families of suicidal adolescents. Finally, the use of a control group gave rise to a problem in only two out of eight projects.

Rejection for one or more reasons was recommended for two of the eight protocols. Five protocols required conditions laid down by an ethical committee to be accepted by the researchers before the research could be approved. One research protocol was fully acceptable in its form as presented.

\section{Discussion}

In this simulation exercise, ERC members reviewing research protocols found many ethical issues to be unresolved in protocols that had been approved by ERCs. Consent was the single most troublesome problem which called for additional safeguards such as written information or the intervention of an independent medical practitioner. The lack of apparent usefulness of the research motivated the rejection of one protocol, and required additional safeguards for two others. The critical view taken of all but one of the research protocols contrasts with the fact that all had already been accepted, in the same form, by institutional ERCs. The more critical attitude in the simulation exercise than in real ERCs could be an indication that institutional ERCs find it difficult to take a sufficiently distant and uninvolved stance with regard to colleagues' proposed research. The inclusion of non-medical members and members from outside the institution has been advocated by many observers and might be supported by this observation.

However, the simulation exercise may well have provided a setting for an over-critical attitude: it was after all carried out during a symposium on ethical problems. Participants may also have chosen to highlight certain problems, knowing that their decisions would have no real effect. There is no reason to believe that the simulation exercise decisions were all right and the original ERC decisions were all wrong. The results cannot therefore be considered as an overall judgement on the effectiveness of ethical review by committee.

What the exercise does highlight is the lack of objective criteria which can define the 'validity' of an ERC decision, given that we have demonstrated a lack of agreement between real and simulated ERCs. Such unreliability is of course a feature of decision-making in medicine as a whole for example in diagnosis, treatment choices and assessments of dangerousness.
This experience does suggest that ERCs could benefit from sharing and comparing their methods of work and evaluative criteria. One way of doing this would be for ERCs to submit regularly a certain proportion of research protocols for which decisions have been reached (for example 10 per cent) to an independent review by another ERC. In return the ERC would receive from its partner protocols for independent review. This form of mutual control and comparison could aid ERCs in defining their criteria, in improving their methods of work and in increasing the credibility of their decision-making.

Any ERC must consider the issues which formed the basis of the simulation exercise: consent, degree of risk and discomfort for research subjects, confidentiality, the use of a control group, the usefulness of the research and follow-up management after the research procedure. The simulated review committees found that the information and criteria needed to make these assessments were often insufficient. No precise evaluative criteria emerge from this exercise but the need for such criteria is clearly felt.

\section{Acknowledgements}

The authors thank Professors Bernheim and Donath (University of Geneva), organisers of the Symposium on Ethics which provided the setting for this study as well as Professor A Mégevand, Dr L Favre and Dr P Schulz, rapporteurs for the three working groups in the simulation exercises.

\section{Authors' note}

This work was done with the informed consent of the original committees.

Timothy Harding is Chargé de cours, and Marinette Ummel is a Research Assistant, at the University Institute of Legal Medicine, Geneva.

\section{References}

(1) Gutteridge F, Bankowski Z, Curran W, Dunne J. The structure and functioning of ethical review committees. In: Bankowski Z, Howard-Jones $\mathrm{N}$, eds. Human experimentation and medical ethics, Geneva: Council for International Organizations of Medical Sciences, 1982.

(2) Allen P, Waters W E. Attitudes to research ethical committees. Fournal of medical ethics 1983; 9:61-65. structure and functioning of ethical review committees.

(3) Lewis P J. The drawbacks of research ethics committees. Fournal of medical ethics 1982; 8:61-64. 